\title{
Time-Decreasing Hazard and Increasing Time until the Next Earthquake
}

\author{
Álvaro Corral* \\ Departament de Física, Facultat de Ciències, Universitat Autònoma de Barcelona, E-08193 Bellaterra, Barcelona, Spain
}

(September 27, 2018)

\begin{abstract}
The existence of a slowly always-decreasing probability density for the recurrence times of earthquakes implies that the occurrence of an event at a given instant becomes more unlikely as time since the previous event increases. Consequently, the expected waiting time to the next earthquake increases with the elapsed time, that is, the event moves away to the future fast. We have found direct empirical evidence of this counterintuitive behavior in two worldwide catalogs as well as in diverse local catalogs. Furthermore, the phenomenon can be well described by universal scaling functions.
\end{abstract}

PACS numbers: 91.30.Dk, 05.65.+b, 89.75.Da, 64.60.Ht

Many probability distributions have been proposed to account for the recurrence time of earthquakes [1, 2, 3, 1 , which is the time interval between successive earthquakes in a certain region. When aftershocks and mainshocks are considered together as a part of essentially one unique process [5, 6], we have determined that a universal scaling law describes the probability density $D(\tau)$ of the recurrence time. In this way, for events of magnitude $M$ above a certain threshold $M_{c}$ in a given spatial area (whose limits do not need to depend on the tectonic background), $D(\tau)$ scales with the rate of seismic activity $R$ in the area as

$$
D(\tau)=R f(R \tau)
$$

where $R$ is defined as the mean number of earthquakes (with $M \geq M_{c}$ ) per unit time and $f$ is a universal scaling function. This scaling is in fact the hallmark of the self-similarity of seismicity in the space-time-magnitude domain. Among several possible scaling functions, the best fit is obtained from a (truncated) gamma distribution,

$$
f(\theta)=\frac{C}{a \Gamma(\gamma)}\left(\frac{a}{\theta}\right)^{1-\gamma} e^{-\theta / a},
$$

with $\gamma$ the shape parameter, $a$ the scale parameter, $C$ a correction to normalization (due to the truncation of the distribution close to zero), and $\Gamma(\gamma)$ the gamma function. When $\gamma<1, f$ turns out to be a decreasing power law, accelerated by an exponential factor in the long-time limit.

We analyze many regions and $M_{c}$-values from two worldwide catalogs (the NEIC-PDE and the Significant Earthquake Database from NOAA at NEIC [7]) and from several regional catalogs (Southern California, Japan, New Zealand, New Madrid (USA), the Iberian Peninsula, and the British Islands [8]). Each analyzed region is delimited by two meridians and two parallels [9], with linear size spanning from $0.16^{\circ}$ (about $18 \mathrm{~km}$ ) to the whole globe $\left(\sim 20 \cdot 10^{3} \mathrm{~km}\right)$, covering a large variety of tectonic environments, whereas the considered magnitudes range from larger than 1.5 to larger than 7.5 (this is about a factor $10^{9}$ in the minimum released energy).

Except for a $360^{\circ} \times 180^{\circ}$-region, which covers the entire globe, the rest of the regions are defined by a window of $L$ degrees in longitude and $L$ degrees in latitude. The coordinates $(x, y)$ of the west-south corner of these regions can be obtained from the vector $\left(k_{x}, k_{y}\right)$ at Fig. 1 's labels as $x=x_{\text {min }}+k_{x} L, y=y_{\text {min }}+k_{y} L$, with $\left(x_{\text {min }}, y_{\text {min }}\right)=\left(-180^{\circ},-90^{\circ}\right),\left(-123^{\circ}, 30^{\circ}\right),\left(127^{\circ}, 27^{\circ}\right)$, $\left(160^{\circ},-60^{\circ}\right),\left(-91^{\circ}, 35^{\circ}\right),\left(-20^{\circ}, 30^{\circ}\right)$, and $\left(-10^{\circ}, 45^{\circ}\right)$ for the worldwide, Southern California, Japan, New Zealand, New Madrid, Iberian Peninsula, and British Island catalogs, respectively. The periods of study are (in years A.D. including the last one) 1973-2002 for the NEIC catalog, 1897-1970 for the NOAA one, 1988-1991, 1995-1998, and 1984-2001 for Southern California (denoted as SC88, SC95, and SC84), and 1995-1998, 19962001, 1975-2002, 1993-1997, and 1991-2001, for the rest of catalogs (in the same order as before). The regions and times of observation are selected in order that a period of stationary seismic activity is included, this means that aftershock sequences should not have too much weight in the seismicity of the region. When this is not the case (i.e., for very large aftershock activity) our analysis is still valid, but the scaling with the mean rate has to be replaced by a scaling with the instantaneous rate.

A maximum-likelihood estimation of the parameters using the rescaled recurrence time $R \tau$ for all the regions and $M_{c}$ 's studied gives $\gamma=0.74 \pm 0.05$, and $a=1.23 \pm$ 0.15 , which yields a coefficient of variation $C V \simeq 1.2$. The constant $C$ is determined from the normalization condition given the minimum value for which the gamma distribution holds; for $\theta>0.05, C=1.10 \pm 0.10$ (see Fig. 1 (a)).

The results of the fit are shown in Fig. 1 (a) using the survivor function, which is defined as $S(\tau) \equiv \operatorname{Prob}\left[\tau^{\prime}>\right.$ $\tau]=\int_{\tau}^{\infty} D\left(\tau^{\prime}\right) d \tau^{\prime}$ (where $\tau^{\prime}$ is a generic label for the recurrence time, while $\tau$ refers to a particular value of 
the same quantity). It is straightforward to obtain that, in our case, $S(\tau)$ should also verify a scaling relation, $S(\tau)=G(R \tau)$, with $G(\theta)=C Q_{\gamma}(\theta / a)$, and $Q_{\gamma}(\theta / a)$ the complement of the incomplete gamma function [10,11. The total agreement between these equations and the measured distributions is clear from the data collapse and the fitting curve in the plot, for intermediate and long recurrence times. The accuracy of the scaling law and the gamma fit is guaranteed as the seismic activity is stationary in this range of recurrence times. On the contrary, short times are usually not free of disturbances of the stationariness, due to the triggering of aftershock sequences, which destroy the universal scaling behavior. In order to treat all the distributions in the same way, we calculate the rate $R$ only for events in the scaling region, i.e., short recurrence times are not considered in the rate.

The knowledge of the probability distribution of the recurrence times allows one to answer two important questions about the temporal occurrence of earthquakes. First, for a certain region and for $M \geq M_{c}$, we can study the probability per unit time of an immediate earthquake given that there has been a period $\tau$ without activity, using the hazard rate [12],

$$
\lambda(\tau) \equiv \frac{\operatorname{Prob}\left[\tau<\tau^{\prime} \leq \tau+d \tau \mid \tau^{\prime}>\tau\right]}{d \tau}=\frac{D(\tau)}{S(\tau)},
$$

where the symbol "|" accounts for conditional probability. From the previous formulas we get that $\lambda(\tau)$ scales as $\lambda(\tau)=R h(R \tau)$, with

$$
h(\theta)=\frac{1}{a \Gamma(\gamma)}\left(\frac{a}{\theta}\right)^{1-\gamma} \frac{e^{-\theta / a}}{Q_{\gamma}(\theta / a)} .
$$

This function turns out to be monotonically decreasing, tending as a power law to the value $1 / a$ as $\theta \rightarrow \infty$. So, contrary to common belief, the hazard does not increase with the elapsed time since the last earthquake, but just the opposite; this is precisely the more direct characterization of long-term clustering [13].

Also, one can wonder about the expected time till the next earthquake, given that a period $\tau_{0}$ without earthquakes (in the spatial area and range of magnitudes considered) has elapsed,

$$
\epsilon\left(\tau_{0}\right) \equiv E\left[\tau-\tau_{0} \mid \tau>\tau_{0}\right]=\frac{1}{S\left(\tau_{0}\right)} \int_{\tau_{0}}^{\infty}\left(\tau-\tau_{0}\right) D(\tau) d \tau .
$$

This function can be referred to as the expected residual recurrence time [12] and again we find a scaling form for it, which is $\epsilon\left(\tau_{0}\right)=e\left(R \tau_{0}\right) / R$, with the scaling function

$$
e(\theta)=a \gamma \frac{Q_{\gamma+1}(\theta / a)}{Q_{\gamma}(\theta / a)}-\theta .
$$

This is an increasing function of $\theta$, which reaches an asymptotic value $e(\theta) \rightarrow a$. Therefore, the residual time until the next earthquake should grow with the elapsed time since the last one. Notice the counterintuitive behavior that this represents: if we decompose the recurrence time $\tau$ as $\tau=\tau_{0}+\tau_{f}$, with $\tau_{f}$ the residual time to the next earthquake, the increase of $\tau_{0}$ implies the increase of the mean value of $\tau_{f}$, but the mean value of $\tau$ is kept fixed. In fact, this is just a more dramatic version of the classical waiting-time paradox 14 15 .

For the particular case of earthquakes this is even more paradoxical, since one would say that the longer the time one has been waiting for an earthquake, the closer it will be, due to the fact that as time passes stress increases on the corresponding fault and the next earthquake becomes more likely. (Nevertheless, one needs to distinguish between earthquakes on a given fault and earthquakes over a certain area.) The question was originally put forward by Davis et al. 16], who pointed out that if a lognormal distribution is a priori assumed for the recurrence times, the expected residual time increases with the waiting time. (However, the increase here was associated to the update of the distribution parameters as the time since the last earthquake, which was taken into account in the estimation, increased; we deal with a different concept of increasing residual time.) Sornette and Knopoff [2] showed that the increase (or decrease) depends completely on the election of the distribution, and studied the properties of a number of them. We are going to see that the observational data provide direct evidence against the simple picture of the next earthquake approaching in time.

Indeed, our mathematical predictions can be contrasted with the catalogs; both the hazard rate and the expected residual recurrence time can be directly measured with no assumption about their functional form. Following their definitions, these quantities are estimated as

$$
\lambda(\tau)=\frac{n(\tau, \tau+\Delta \tau)}{n(\tau, \infty) \Delta \tau}, \quad \epsilon\left(\tau_{0}\right)=\frac{\sum_{i \mid \tau_{i}>\tau_{0}}\left(\tau_{i}-\tau_{0}\right)}{n\left(\tau_{0}, \infty\right)},
$$

where $n\left(\tau_{1}, \tau_{2}\right)$ denotes the number of quakes with recurrence time in the range $\left(\tau_{1}, \tau_{2}\right)$ and the sum in $\epsilon\left(\tau_{0}\right)$ is computed only for earthquakes $i$ such that $\tau_{i}>\tau_{0}$ (and of course $M \geq M_{c}$ ). From the results displayed in Figs. 1 (b) and 1 (c) it is apparent that the hazard rate decreases with time whereas the expected residual recurrence time increases, as we predicted. Moreover, both quantities are well approximated by the proposed universal scaling functions.

On the other hand, the part of the recurrence-time distribution that accounts for short times displays a typical behavior $f(\theta)=K_{1} / \theta^{1-\alpha}$, and the corresponding functions for the survivor function, hazard rate, and expected residual return time turn out to be:

$$
G(\theta)=K_{1}\left(K_{2}-\theta^{\alpha} / \alpha\right), \quad h(\theta)=\frac{1}{K_{2} \theta^{1-\alpha}-\theta / \alpha},
$$




$$
e(\theta)=\frac{K_{3}-\theta^{1+\alpha} /(1+\alpha)}{K_{2}-\theta^{\alpha} / \alpha}-\theta,
$$

where the constants $K_{2}$ and $K_{3}$ depend on the rest of the distribution. An example for these functions with $\alpha \simeq$ 0.2 is also represented in Fig. 1, showing the appropriate decreasing or increasing tendency in each case.

For the sake of concreteness, let us consider worldwide earthquakes with $M \geq 7.5$, which occur at a rate $R=6$ per year, roughly. In the days immediately after one event of this type, the expected time to the next one (anywhere in the world) is about 2 months (for $\tau=6$ days, we have $R \tau=0.1$, and $e(0.1) \simeq 1$, see Fig. 1 (c)). If after 2 months the quake has not come, the expected residual time not only does not decrease but increases to 2.2 months $(e(1) \simeq 1.1$, this would lead to 4.2 months between both events), and if the elapsed time reached 1 year (which is unlikely but not impossible), the expected waiting time would further increase to 2.4 months $(e(6) \sim 1.2)$. In the same way, the hazard rate would drop from 0.7 to 0.5 and to 0.4 month $^{-1}(h(\theta) \simeq 1.4,1$, and 0.85$)$, respectively. The same process is reproduced at all magnitude and spatial scales in a self-similar manner. An intuitive explanation of this phenomenon is that when the elapsed time since the last earthquake is large, the system enters into a long "drought period" in which the recurrence time is likely to be very large. Notice however that there is no fundamental difference between these drought periods and the rest of recurrence times, since all of them are governed by the same smooth distribution.

The universality of this behavior demands further explanation; nevertheless, it suggests the existence of a simple mechanism in which, as time passes, the variable that triggers rupture runs away from the rupture threshold (on average). The "excursions" of this variable would keep the memory of the last event stored in the system up to very long times to generate the negative aging observed.

The considerations reported here should be at the core of any research regarding earthquake-occurrence modeling [17, 18 and predictability [19,20,21,22. Finally, in order to account for the self-similarity of these processes, the concept of self-organized criticality provides the most appealing framework up to now 23.

This author has benefited a lot from the original perspectives and deep insights of the late Per Bak. The author also thanks M. Boguñá, D. Sornette's criticisms, the Ramón y Cajal program of the Spanish MCyT, and all the people at the Statistical Physics Group of the Universitat Autònoma de Barcelona, as well as those institutions that have made their catalogs available on the Internet.
* E-mail address: Alvaro.Corral@uab.es

[1] R. F. Smalley, J.-L. Chatelain, D. L. Turcotte, R. Prévot, Bull. Seism. Soc. Am. 77, 1368 (1987).

[2] D. Sornette, L. Knopoff, Bull. Seism. Soc. Am. 87, 789 (1997).

[3] J.-H. Wang, C.-H. Kuo, J. Seism. 2, 351 (1998).

[4] W. L. Ellsworth et al., "A physically-based earthquake recurrence model for estimation of long-term earthquake probabilities" (U.S. Geological Survey Open-File Report 99-522, 1999).

[5] P. Bak, K. Christensen, L. Danon, T. Scanlon, Phys. Rev. Lett. 88, 178501 (2002).

[6] K. Christensen, L. Danon, T. Scanlon, P. Bak, Proc. Natl. Acad. Sci. USA 99, 2509 (2002).

[7] National Earthquake Information Center, http://wwwneic.cr.usgs.gov/neis/epic/epic_global.html

[8] Southern California $\quad$ Seismographic Network, http://www.scecdc.scec.org/ftp/catalogs/SCSN; Japan University Network Earthquake Catalog, http://wwweic.eri.utokyo.ac.jp/CATALOG/junec/monthly.html;

GeoNet, http://www.geonet.org.nz/eq-search.jsp; Center for Earthquake Research and Information, http://folkworm.ceri.memphis.edu/catalogs/html/cat_nm.htm; Instituto Geográfico Nacional, Boletín de Sismos Próximos, http://www.geo.ign.es/servidor/sismo/cnis/terremotos.htm; British Geological Survey, catalog available upon request.

[9] Except of course for regions ending at the poles.

[10] M. Abramowitz, I. A. Stegun, Eds., Handbook of Mathematical Functions (Dover, New York, 1965).

[11] W. H. Press, S. A. Teukolsky, W. T. Vetterling, B. P. Flannery, Numerical Recipes in FORTRAN (Cambridge University Press, Cambridge, ed. 2, 1992).

[12] J. D. Kalbfleisch, R. L. Prentice, The Statistical Analysis of Failure Time Data (Wiley, New York, 1980).

[13] Y. Y. Kagan, D. D. Jackson, Geophys. J. Int. 104, 117 (1991).

[14] W. Feller, An Introduction to Probability Theory and Its Applications (Wiley, New York, ed. 2, 1971), vol. 2.

[15] M. Schroeder, Fractals, Chaos, Power Laws (Freeman, New York, 1991).

[16] P. M. Davis, D. D. Jackson, Y. Y. Kagan, Bull. Seism. Soc. Am. 79, 1439 (1989).

[17] Y. Ogata, Pure appl. geophys. 155, 471 (1999).

[18] A. Helmstetter, D. Sornette, Phys. Rev. E 66, 061104 (2002).

[19] I. Main, Nature 385, 19 (1997).

[20] R. J. Geller, D. D. Jackson, Y. Y. Kagan, F. Mulargia, Science 275, 1616 (1997).

[21] Nature Debates, "Is the reliable prediction of individual earthquakes a realistic scientific goal?" http://www.nature.com/nature/debates/index.html

[22] T. Parsons, S. Toda, R. S. Stein, A. Barka, J. H. Dieterich, Science 288, 661 (2000).

[23] P. Bak, How Nature Works: The Science of Self-Organized Criticality (Copernicus, New York, 1996). 

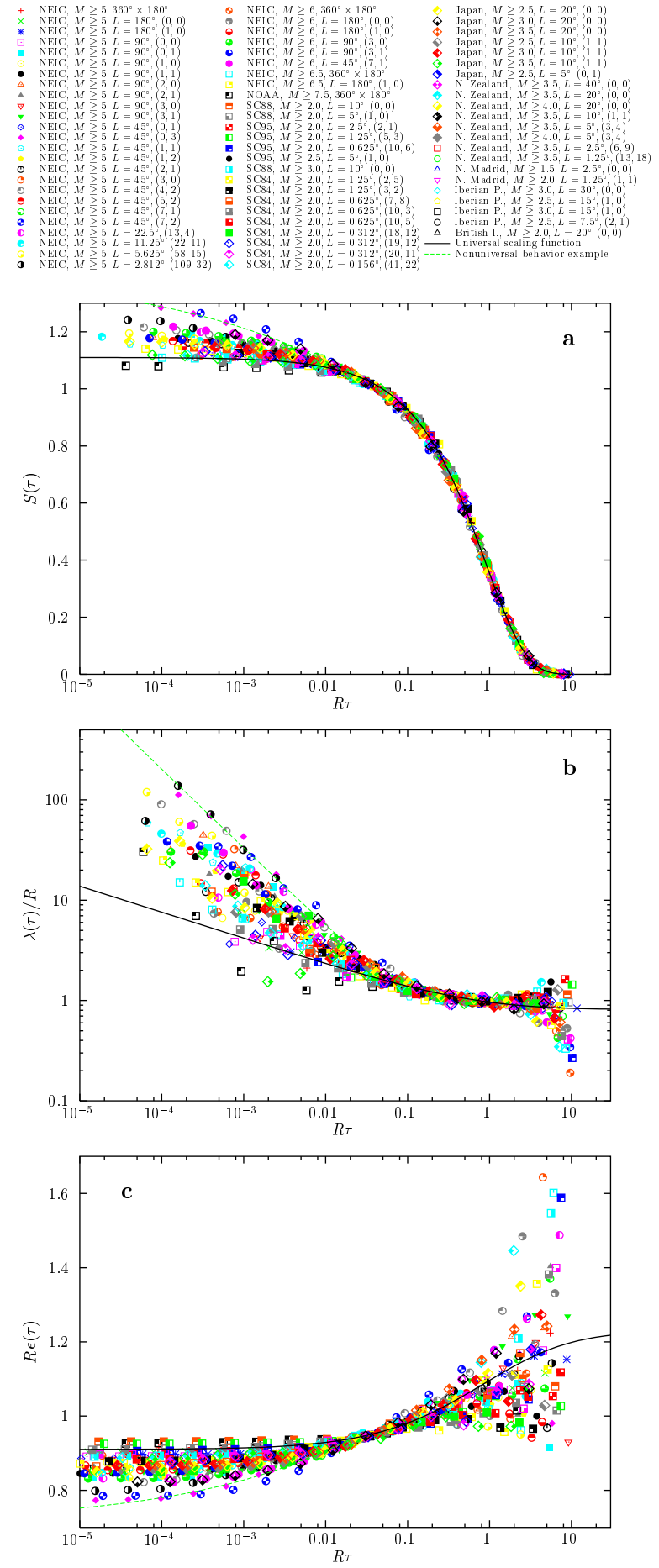

FIG. 1. Scaling plots of the probability distributions, hazard rate functions, and expected residual recurrence times for all the catalogs analyzed. The values of the occurrence rates $R$ are broadly distributed, ranging roughly from 6 year $^{-1}$ to 1 hour $^{-1}$, so one unit in the horizontal axis can represent from 1 hour to 2 months. The universal scaling functions fitting the data are the ones proposed in the text, with the parameters obtained from the maximum-likelihood estimation; an example of fit outside the scaling region is also shown. (a) Rescaled survivor functions. The distributions are normalized for $R \tau \geq 0.05$, therefore, the left part of the distributions does not represent a probability; nevertheless, we have considered interesting to show it to illustrate the nonuniversal behavior. Times shorter than two minutes are not shown. (b) Rescaled hazard rates. The errors at long times are large, due to poor statistics. (c) Rescaled expected residual recurrence times. Only mean values calculated with 3 or more data are displayed. At long times the errors increase even further in this case, as $\epsilon(\tau)$ is the difference of two large numbers; nevertheless, the gaps between the points and the function are compatible with the error bars (not shown). 